\title{
Percepções de acadêmicos de educação física do sul do Brasil sobre o tema transversal saúde
}

\author{
Perceptions of academic physical education in southern Brazil \\ on the theme cross health

\section{Percepciones de académico de educación física en el sur de Brasil sobre el tema de la cruz de la salud}

\author{
Cati Reckelberg AZAMBUJA ${ }^{1,2}$ \\ Kelly Christine Maccarini PANDOLFO ${ }^{2}$ \\ Rafaella Righes MACHADO ${ }^{2}$ \\ Daniela Lopes dos SANTOS ${ }^{1,2}$ \\ Maria Rosa Chitolina SCHETINGER ${ }^{3}$
}

\footnotetext{
${ }^{1}$ Programa de Pós-Graduação em Educação Física, Universidade Federal de Santa Maria - UFSM, 97.105-900, Santa Maria - RS, Brasil ${ }^{2}$ Núcleo de Estudos em Exercício Físico e Saúde, Universidade Federal de Santa Maria - UFSM, 97.105-900, Santa Maria - RS, Brasil ${ }^{3}$ Programa de Pós-Graduação em Educação em Ciências: Química da Vida e Saúde, Universidade Federal do Rio Grande do Sul - UFRGS, 90.035-003, Porto Alegre - RS, Brasil
}

\begin{abstract}
Resumo
A dificuldade dos professores em contextualizar o saber tem origem no processo de formação inicial e se reflete na produção e transmissão dos conhecimentos. O objetivo do presente estudo foi investigar as concepções de acadêmicos de licenciatura em educação física, a respeito do entendimento sobre a educação em saúde e dos Temas Transversais Saúde na escola. Participaram da pesquisa 16 acadêmicos de Educação Física. Utilizou-se um questionário composto por dez questões, abertas e fechadas, sobre o Tema Transversal Saúde e a educação em saúde no ambiente escolar. Os resultados apontaram que os licenciandos de educação física possuem conhecimento restrito sobre educação em saúde e sua abordagem junto aos alunos, demonstrando por suas respostas que estes podem ter sido induzidos pelo senso comum, que preconiza a indispensável condição de boa qualidade de vida e manutenção de saúde.
\end{abstract}

Descritores: Educação em Saúde; Educação Física, Serviços de Saúde Escolar.

\begin{abstract}
The difficulty of teachers to contextualize knowledge originates from the initial training process and is reflected in the production and transmission of knowledge. The aim of this study was to investigate the concepts of academic degree in physical education, about the understanding of health education and Transversal Themes Health in school. 16 students participated in the survey of Physical Education. We used a questionnaire consisting of ten questions, open and closed, on the Cross Tema Health and health education in the school environment. The results showed that the physical education student teachers have limited knowledge about health education and their approach to the students, demonstrating by their responses that they may have been induced by common sense, which advocates the indispensable condition of good quality of life and maintaining health.
\end{abstract}

Descriptors: Health Education; Physical Education; School Health Services.

\section{Resumen}

La dificultad de los profesores para contextualizar el conocimiento se origina en el proceso de formación inicial y se refleja en la producción y transmisión de conocimiento. El objetivo de este estudio fue investigar los conceptos de grado académico en educación física, sobre la comprensión de la educación para la salud y temas transversales de salud en la escuela. 16 estudiantes participaron en la encuesta de Educación Física. Se utilizó un cuestionario que consta de diez preguntas, abiertas y cerradas, en Tema Transversal Salud y educación para la salud en el entorno escolar. Los resultados mostraron que los futuros profesores de educación física tienen un conocimiento sobre educación para la salud y su acercamiento a los estudiantes, lo que demuestra por sus respuestas limitadas que pueden haber sido inducidos por el sentido común, que aboga por la condición indispensable de la buena calidad de vida y el mantenimiento de salud.

Descriptores: Educación para la Salud; Educación Física; Servicios de Salud Escolar. 


\section{INTRODUÇÃO}

A Educação Brasileira reflete as consequências dos movimentos de globalização que, no decorrer das últimas décadas, vem gerando profundas e complexas transformações, desde a estrutura organizacional das Instituições de Ensino e de seus objetivos de educação, até a atuação dos professores ${ }^{1}$, objetivando o desenvolvimento da capacidade dos alunos para atuarem como cidadãos ativos na sociedade ${ }^{2}$. Segundo Reis ${ }^{3}$, somente a interdisciplinaridade poderá oferecer eficiência e autonomia a um sistema educativo, proporcionando uma educação de qualidade, em que os conteúdos ensinados não sejam apresentados de maneira compartimentalizada e, desta forma, diminuindo o engavetamento e o isolamento dos mesmos em relação ao contexto.

Na tentativa de evitar este tipo de situação, a saúde na escola vem sendo desenhada durante uma série de encontros mundiais ${ }^{4}$ e, a partir disso, o Ministério da Educação e do Desporto ${ }^{5}$ criou o referencial curricular nacional, no qual a saúde foi considerada como um tema transversal a ser trabalhado com responsabilidade no projeto de toda a escola, o que torna alunos, professores e o próprio ambiente escolar, sistematicamente, elementos chaves para essa realização.

O desenvolvimento e a abordagem dos Temas Transversais (TT), especificamente o Tema Transversal Saúde (TTS), só torna-se possível e eficiente diante de uma proposta de interdisciplinaridade e a contextualização dos assuntos pautados, permitindo que ocorra a transposição didática entre os conteúdos, ao mesmo tempo em que se encontra alicerçado em princípios curriculares que se complementam e contribuem para que o aluno compreenda a realidade como um complexo sistema. Hartmann e Zimmermann ${ }^{6}$, afirmam que os alunos, ao estudarem os fenômenos com uma abordagem sistêmica, estimulam a organização do pensamento e do estudo pela análise e pela síntese. Desta maneira, eles têm a possibilidade de construir um conhecimento integrado, além de organizar seu pensamento para que estabeleçam as ligações, assim como, saibam diferenciar os saberes.

Neste contexto, as escolas apresentam-se como espaços privilegiados, pois são capazes de unir, em só ambiente, tanto professores, como alunos, permitindo o cumprimento de vários papéis. Contudo, apesar do TTS integrar os conteúdos propostos pelos Parâmetros Curriculares Nacionais (PCN), a abordagem realizada pelos professores ainda é limitada ${ }^{7}$, pressupondo-se advir de uma formação inicial insuficiente ${ }^{8}$.

Diante do referido anteriormente, estabelece-se o desafio de superar os problemas advindos de uma formação inicial precária e potencializá-las como oportunidades de estruturação da base de conhecimento, assim como da construção do saber pedagógico dos futuros professores ${ }^{9}$ para que a abordagem dos conteúdos referentes a saúde não se limite ao caráter eminentemente biológico e informativo ${ }^{10}$. Sendo assim, o objetivo deste estudo foi investigar as concepções de acadêmicos de licenciatura do curso de educação física, em fase final de curso, a respeito do entendimento sobre a educação em saúde e do TTS na escola.

\section{MATERIAL E MÉTODO}

O estudo foi realizado com corte transversal e possui caráter quantitativo, pois utiliza vários casos e um número reduzido de variáveis, assim como, também apresenta característica qualitativa do tipo descritiva, por ter como objetivo principal a descrição das características de determinada população na tentativa de buscar a compreensão e o significado das mesmas ${ }^{11}$.

Participaram do estudo 16 acadêmicos (alunos de curso de nível superior) em fase final de formação $\left(7^{\circ}\right.$ semestre) do curso de Licenciatura em Educação Física de uma Universidade Federal do interior do Sul do Brasil, os quais receberam instrução a respeito da pesquisa quanto à preservação de identidade e prestação de esclarecimentos em caso de dúvida, sendo solicitado que assinassem o Termo de Consentimento Livre e Esclarecido (TCLE). O presente estudo foi aprovado pelo Comitê de Ética, segundo a Resolução no 196/96 ${ }^{12}$, sob o protocolo de Certificado de Apresentação para Apreciação Ética (CAAE) no 0094.0.243.000-11.

Para a coleta dos dados utilizou-se como instrumento questionário semi-estruturado, composto por dez questões, abertas e fechadas, que abrangeram desde a autopercepção em relação ao entendimento do desenvolvimento dos temas transversais e da educação em saúde no ambiente escolar, assim como a respeito da formação acadêmica para a aplicação da educação em saúde nos conteúdos da educação física escolar (Tabela 1). Os instrumentos aplicados aos acadêmicos (questionários) foram coletados no mês junho de 2013.

A construção de um instrumento foi necessária para poder atender as necessidades investigativas do estudo, especificamente, no que diz respeito às questões que versam sobre como o acadêmico de Educação Física estaria se sentindo no que se refere ao seu preparo para atuar na escola através da abordagem interdisciplinar de temas relacionados à saúde. Desta forma, após a elaboração das questões, foi realizado um Teste Piloto do instrumento em um grupo de alunos de Educação Física com características semelhantes às da pesquisa, mas que não participaram da amostra final, com o objetivo de testar e realizar adequações.

Além disso, o questionário foi submetido à Validação através da análise de sua Matriz Analítica por três especialistas da área. Foram sugeridas correções, as quais foram acolhidas e, após a reformulação do instrumento e retorno aos professores, foi emitido parecer favorável para a sua utilização nesta pesquisa. 
Tabela 1. Questões aplicadas aos acadêmicos de educação física

\begin{tabular}{l} 
Questões \\
\hline 1 - O que você entende por temas transversais na educação? \\
2 - O que você entende por educação em saúde? \\
3 - O currículo do seu curso de graduação aborda a questão da educação \\
em saúde na escola? \\
4 - Durante a graduação, você teve ou sabe que terá alguma prática de \\
ensino que aborde aspectos referentes à educação em saúde? \\
5 - Qual a sua visão em relação ao papel da escola na educação em saúde \\
dos alunos? \\
6- Quem você considera que seja o responsável pela educação em saúde \\
na escola? \\
7 - Você se considera preparado para desenvolver o tema transversal \\
saúde na escola? \\
8- Em quais conteúdos do plano de ensino você abordaria os temas \\
relacionados à saúde? \\
- Você considera que os conteúdos ensinados na educação física escolar \\
ajudam os alunos a viver melhor? \\
10 - Como você considera a atuação dos professores de educação física em \\
relação à educação em saúde?
\end{tabular}

Os dados foram categorizados conforme a Análise de Conteúdo ${ }^{13,14}$. Para a Análise de Conteúdo seguiramse alguns passos preconizados pela literatura pertinente quais sejam: a) leitura flutuante; b) desconstrução e unitarização dos textos; c) categorização; d) construção de um metatexto com expressão das compreensões atingidas.

\section{RESULTADOS E DISCUSSÃO}

Os acadêmicos do curso de Educação Física foram questionados a respeito da sua formação para a abordagem dos TT na escola, especificamente o TTS, assim como a visão destes futuros professores sobre a importância da inserção destes conteúdos nas aulas de Educação Física.

Inicialmente, ao ser perguntado para os acadêmicos a respeito do seu entendimento dos TT na educação, a maioria destes afirmaram que estes são considerados outros temas que não àqueles da própria disciplina de Educação Física, como pode ser evidenciado na resposta de um dos alunos: “[...]são outros conteúdos além daqueles próprios da Educação Física", demonstrando que os mesmos não possuem um conhecimento básico, necessário para a discussão da temática junto aos alunos. Mais preocupante ainda, foram os que afirmaram que os TT são os conteúdos específicos da Educação Física, como por exemplo, "[...] esporte, dança, lutas", ou ainda, "[...]assuntos que estão sendo veiculados na mídia".

Segundo Loguercio e Del Pino ${ }^{15}$, a problemática envolvendo a dificuldade dos professores em contextualizar o conhecimento é antiga e está condicionada apenas na transmissão dos conhecimentos, ao passo que deveriam ser produtores destes. Esta prática docente encontra-se presente igualmente no ensino superior, o que acaba por refletir expressivamente na formação dos acadêmicos, como pode ser evidenciado no parágrafo anterior.

Avançando nos questionamentos, os alunos foram indagados sobre o entendimento em Educação em Saúde. A grande maioria afirmou que a Educação em Saúde compreende as questões relacionadas ao estilo e a qualidade de vida saudável, além de ser uma ferramenta para a Educação em Saúde, como demonstradas nas falas de dois acadêmicos: "[...]educar para formar alunos críticos com questões relacionadas a saúde" e "[...]estabelecer relações de temas da saúde na abordagem de conteúdos educacionais”.

Identifica-se, pelas respostas apresentadas nas questões anteriores, que os alunos compreendem a importância de abordagem dos assuntos relacionados à saúde em ambiente escolar, porém, não conseguem estabelecer uma transversalidade do tema saúde nos componentes curriculares da disciplina. As diretrizes dos $\mathrm{PCN}^{5}$ determinam que o ensino estabeleça comunicação entre a informação científica e o contexto social, instituindo a perspectiva interdisciplinar e contextualização dos conhecimentos. A interdisciplinaridade busca a resolução de problemas concretos ou compreensão de fenômenos sob diferentes pontos de vista através da utilização do conhecimento de várias disciplinas, aproximando o saber científico das questões sociais contemporâneas.

A formação inicial de professores de educação física tem sido questionada por diversos autores ${ }^{16-18}$. Sendo assim, as questões seguintes trouxeram a problematização acerca desta formação dos futuros professores de Educação Física em relação à inserção do TTS e suas abordagens na grade curricular do curso de graduação.

Foram encontradas algumas ocorrências de que o TTS não foi trabalhado durante o curso de graduação até o momento. Contudo, mesmo a maioria dos alunos respondendo "sim", as respostas foram complementadas com falas que expressavam a carência deixada pelo assunto quando abordado nas aulas da graduação: "[...]muito fragmentada", "[...]pouco abordado", "[...]pouquíssimo", "[...]apenas com reflexões sobre o assunto", demonstrando uma importante falha que o currículo apresentou, explorando de forma insuficiente a temática.

Sobre a prática de ensino que abordasse aspectos referentes à Educação em Saúde durante a graduação, os acadêmicos relataram que quando trabalhados os assuntos relacionados à Educação em Saúde, estes foram desenvolvidos nas aulas das disciplinas de Educação em Saúde, Fisiologia, Urgência e Emergência, Cinesiologia, Equoterapia e Laboratório II.

Segundo Lovisolo ${ }^{19}$, ao longo dos anos, os cursos de licenciatura em Educação Física vêm sendo descaracterizados por falta de uma identidade profissional, além da ausência de um objeto de estudo claro para área, tornando-se um limitador para o que determinam os PCN em relação à saúde, É sabido que apesar da proposta dos PCN recomendar a transversalidade entre os conteúdos, muitos professores que atuam nas escolas não tiveram, em suas formações universitárias, os conteúdos dos TT. Além disso, segundo Darido et al., "há a questão da tradição que 
acompanha cada área, o que dificulta a incorporação de outras maneiras para se trabalhar com os conteúdos"20.

Neste contexto, destacado o papel que é desempenhado pelos professores que atuam diretamente com os alunos, contribuindo para a sua formação e, que necessitam capacitações e atualizações em promoção da saúde. Segundo Copetti et al. ${ }^{21}$, o ensino da saúde tem sido um desafio para a educação no que se refere à possibilidade de garantir uma aprendizagem efetiva e transformadora de atitudes e hábitos de vida, pois estes hábitos e atitudes, adquiridos na infância e na adolescência, influenciam diretamente o padrão de comportamento na fase adulta.

Mohr $^{22}$ faz um alerta quanto ao desenvolvimento e a responsabilidade da educação em ciências no ambiente escolar, afirmando que esta, normalmente, está incumbida aos professores da disciplina de Ciências, especialmente os de Ciências Biológicas. Sendo assim, os futuros professores foram questionados sobre o papel da escola e a quem deve ser atribuída a função de educar em saúde no ambiente escolar. Muitos deles afirmaram entender a importância da abordagem da temática saúde no contexto escolar, mas não especificaram de que forma isso pode acontecer, como pode ser evidenciado em respostas como: "[...]papel fundamental", "[...]deve ser trabalhado", "[...]deve ser abordado". Além disso, alguns outros quem?, definiram que a escola aparece com o papel intervencionista, como demonstrado nas respostas: "[...]conscientizar o aluno a ter hábitos saudáveis de alimentação e prática de atividades físicas", "[...]orientar sobre hábitos de saúde e higiene" e "[...]incentivar hábitos saudáveis". Torna-se importante destacar a visão de alguns dos acadêmicos a respeito da abordagem interdisciplinar, onde, segundo eles, os temas deveriam ser abordados e compartilhados de maneira interdisciplinar. Ainda, faz-se o registro de um aluno que respondeu ao questionamento afirmando: "[...]disponibilizar um profissional da saúde".

Em relação a quem caberia a responsabilidade da Educação em Saúde na escola, houve unanimidade entre os acadêmicos que citaram o professor de Educação Física como o responsável por esta tarefa. Ainda, alguns inquiridos demonstraram em suas respostas que entendem que esta também é uma função dos demais professores envolvidos no processo educacional e, um deles atribui a função a toda comunidade escolar.

Historicamente, as ações educativas em saúde eram exclusivamente de caráter informativo, priorizando a prevenção às doenças, em detrimento de outras dimensões, como a social, a psicológica, a afetiva e a cultural $^{23}$. Esta visão encontra-se cristalizada na prática de alguns profissionais da educação ${ }^{24}$, assim como, na visão de alguns adolescentes ${ }^{25}$ que poderão transpor para a vida adulta esta forma de pensar, interferindo de maneira negativa nas próximas gerações.
Segundo Leonello e L'Abbate ${ }^{26}$, esta maneira de pensar exclui o sujeito do processo educativo, tornandoo um "objeto passivo e aquém da construção reflexiva sobre suas condições de vida e saúde". No mesmo estudo, os autores apontaram um conjunto de possibilidades que podem promover a mudança de comportamento e de atitudes dos alunos em relação à saúde, destacando a necessidade de utilização de estratégias pedagógicas como as discussões, orientações, palestras, aulas e campanhas, assim como, a melhor qualificação dos professores, desde a formação inicial até a continuada.

Desta forma, foi uma preocupação deste estudo, investigar as percepções dos licenciandos em Educação Física, já em fase de conclusão da formação inicial, sobre o quão se sentem preparados para desenvolver o TTS na escola e, ainda, em quais conteúdos do plano de ensino da disciplina de Educação Física, poderiam abordar este tema.

A grande maioria dos acadêmicos admitiu não haver preparo suficiente durante a formação inicial e percebem-se vulneráveis quanto à necessidade de abordagem do TTS durante as aulas. Ressalta-se que um dos acadêmicos afirmou que teria condições de desenvolver a temática porque "[...]é uma temática que está em alta". E, outro que acredita estar preparado, ao responder, "Sim, pois conheço os benefícios da atividade física". No grupo estudado, apenas dois acadêmicos colocaram que se sentiam preparados, com a justificativa de ter sido disponibilizado este conhecimento entre os conteúdos, durante a graduação.

Sendo assim, surge o questionamento: como o licenciando irá conseguir desenvolver o TTS no contexto escolar se, durante a sua formação, não foi abordada e nem discutida a inserção desses nos conteúdos específicos da Educação Física? Está evidente que existe a necessidade de uma transformação da prática docente dos professores das instituições de ensino superior, responsáveis pela formação inicial de futuros professores, pois para Eichler e Del Pino" ${ }^{27}$, "a experiência científica destes professores é descolada de sua docência", motivada pela sua própria formação inicial, o que desencadeia um círculo vicioso do processo.

Em relação ao plano de ensino, enquanto boa parte dos alunos respondeu que seria adequado abordar o TTS em todos os conteúdos, três respostas se referiram a nenhum conteúdo específico. Também foram verificadas respostas que relacionavam a saúde à prática desportiva e à dança. Diante do fato da falta de consenso em alguns pontos, os conteúdos de saúde podem ser considerados complexos. Em sua análise, Knuth et al. ${ }^{10}$ apontam três eixos teóricos que representam estas inadequações: tratamento superficial dos conceitos de saúde, restringindo-o ao aspecto biofísiológico; ênfase na 
abordagem teórica dos conceitos, impedindo a intervenção direta com os alunos; divisão e fortalecimento do rompimento entre conhecimento biológico e das Ciências Humanas. Ainda, segundo os autores, na tarefa de debater a saúde no ambiente escolar "entra em choque uma série de tradições e de discursos muitas vezes conflitantes", sugerindo que uma reformulação curricular na formação dos professores de Educação Física poderá ajudar a modificar este quadro.

Diversos estudos tem demonstrado a preocupação do meio acadêmico em relação às formas de abordagem do TTS na escola ${ }^{23,28-30}$. Nesse sentido, Copetti et al. ${ }^{21}$ afirmam que seria pertinente que as diversas disciplinas se articulassem em um trabalho conjunto, buscando que a educação em saúde estimule a aquisição de conhecimentos relacionados à saúde, o que pode garantir aos envolvidos no processo uma maior preocupação com sua qualidade de vida.

Sobre a importância dos conteúdos e a transposição destes para ajudar os alunos a viverem melhor, as respostas se direcionaram positivamente para aquisição de hábitos saudáveis, como pode ser observado na seguinte afirmação: [...]"Sim, porque incentiva $o$ aluno a praticar a atividade física dentro e fora da escola". Porém, alguns dos acadêmicos parecem ter respondido equivocadamente ao afirmarem: "Não, porque só trabalha o esporte, que os conteúdos são sistematizados dentro esporte".

Identifica-se, claramente, que as respostas carregam fortemente a visão de desenvolvimento do esporte enquanto conteúdo da Educação Física, onde o aprendizado se restringe ao gesto técnico de modalidade específica, caracterizado como uma abordagem tecnicista. Esta evidência nos permite avaliar que os acadêmicos estão com o campo de perspectivas limitado para a Educação Física, ao não conseguirem visualizar as reais capacidades que a disciplina tem a oferecer, sendo que esta objetiva, também, a incorporação de bons hábitos de vida, como os da prática de atividade física e da alimentação adequados, do controle de estresse, dos relacionamentos intra e interpessoais e de comportamentos preventivos, demonstrando que os vários objetivos podem ser trabalhados inclusive durante as práticas desportivas.

Por fim, foi perguntado sobre a importância da atuação dos professores de Educação Física para a Educação em Saúde. Todos consideraram que a atuação do professor de Educação Física é importante e indispensável. Apesar desta afirmação, essas respostas mostraram-se contraditórias em relação aos questionamentos anteriores, pois foi observada grande dificuldade em responder adequadamente as questões que diziam respeito ao conhecimento sobre educação em saúde e sua abordagem junto aos alunos, demonstrando que os acadêmicos possuem precário entendimento sobre o tema.
Ao que parece, os mesmos foram levados pelo senso comum ao afirmar que a Educação em Saúde mostra-se relevante e importante diante dos apelos sociais lançados sobre a indispensável condição de boa qualidade de vida e manutenção de saúde. Além disso, destaca-se o importante papel da universidade frente à formação dos futuros professores de Educação Física, neste caso específico, em relação ao TTS.

\section{CONCLUSÃO}

Como reflexão, propõem-se discutir as diferentes esferas desta problemática, a qual envolve a conexão entre a produção do conhecimento em meio acadêmico e sua aplicação no contexto escolar brasileiro que, em suas políticas públicas educacionais, prevê a inserção dos TT no ensino fundamental ( $1^{\circ}$ ao $9^{\circ}$ Anos) e, a reavaliação do professor enquanto a dimensão do seu ato de ensinar, assumindo postura crítica no pleno exercício de suas atividades.

Destaca-se que a necessidade de programas intervencionistas que abordem as questões como boa alimentação, bons níveis de atividade física, combate ao estresse e mais horas de lazer ativo, consideradas de primordial relevância à saúde das pessoas, deve priorizar o combate à obesidade e ao sedentarismo, esclarecendo e fornecendo subsídios para que os indivíduos despertem para a aquisição de uma boa qualidade de vida, buscando saúde e longevidade.

Por fim, cabe registrar que o estudo apresentou algumas limitações, principalmente em relação à amostra. Como os dados aqui apresentados fizeram parte de uma pesquisa maior, do tipo guarda-chuva, que avaliou o TTS na esfera pública federal de uma cidade do interior do Sul do Brasil, a seleção da amostra se deu de forma intencional e foi delimitada aqueles acadêmicos de Educação Física da única instituição pública federal da cidade que se encontravam em fase de conclusão do curso.

Este foi um critério adotado, pois se entende que somente próximo do final da graduação, eles sejam capazes de demonstrar uma visão coerente com os conhecimentos que acumularam no decorrer da licenciatura, o que poderia influenciar diretamente no resultado desta pesquisa, levando-se em consideração que o principal objetivo era o de investigar as concepções de acadêmicos de licenciatura do curso de educação física, a respeito do entendimento sobre a educação em saúde e do TTS na escola.

\section{REFERÊNCIAS}

1. Matos M, Sardinha LS. Estilos de Vida Activos e Qualidade de Vida. In: Sardinha L, Matos M, Loureiro I (Eds.), Promoção da Saúde: modelos e práticas de intervenção nos âmbitos da actividade física, nutrição e tabagismo. Lisboa: FMH; 1999. 
2. Coutinho RX, Santos WM, Folmer V, Rocha JBT, Puntel RL. Percepções de professores de ciências, matemática e educação física sobre suas práticas em escolas públicas. Rev Ciências \& Ideias. 2012; 4(1):1-18.

3. Reis MBF. Interdisciplinaridade na prática pedagógica: um desafio possível. Revelli. 2009; 1(2):26-45.

4. Fernandes MH, Rocha VM, Souza DB. A concepção sobre saúde do escolar entre professores do ensino fundamental ( $1^{\mathrm{a}}$ a $4^{\mathrm{a}}$ séries $)$. Hist cienc saudeManguinhos. 2005; 12(2):283-91.

5. Brasil. Secretaria de Educação Fundamental. Parâmetros Curriculares Nacionais: ciências naturais. Brasília: MEC/SEF 1997, 136 p. Disponível em: http://portal.mec.gov.br/seb/arquivos/pdf/livro04.pdf. Acesso em: 12 jan2014.

6. Hartmann ÂM, Zimmermann E. Feira de ciências: a interdisciplinaridade e a contextualização em produções de estudantes de ensino médio. In: Anais do VII Encontro Nacional de Pesquisa em Educação em Ciências - ENPEC; Florianópolis: ABRAPEC, 2009.

7. Galindo CJ, Inforsato EC. Manifestações de necessidade de formação continuada por professores do $1^{\mathrm{o}}$ ciclo do ensino fundamental. Dialogia. 2008; 7(1):63-76.

8. Cordeiro OLC, Souza WL. A formação continuada do professor do ensino fundamental de $1^{\mathrm{a}}$ à $4^{\mathrm{a}}$ série na perspectiva da LDB 9.394/96 [trabalho de Conclusão de Curso]. Belém: Universidade da Amazônia; 2002.

9. Marcon D, Graça ABS, Nascimento JV. Critérios para a implementação de práticas pedagógicas na formação inicial em educação física e implicações no conhecimento pedagógico do conteúdo dos futuros professores. Rev bras educ fís esporte. 2011; 25(3):497-511.

10. Knuth AG, Azevedo MR, Rigo LC. A inserção de temas transversais em saúde nas aulas de educação física. RBAFS. 2007; 12(3):73-78.

11. Gil AC. Métodos e Técnicas de Pesquisa Social. São Paulo: Atlas, 1999.

12. Brasil. Ministério da Saúde. Conselho Nacional da Saúde. Resolução no 196 de 10 de outubro de 1996. Brasília: MS/CNS/CONEP. 1996. Disponível em: http://conselho.saude.gov.br/resolucoes/1996/reso19 6.doc. Acesso em 7 fev2014.

13. Bardin L. Análise de Conteúdo. $3^{\mathrm{a}}$ ed. Lisboa: Edições 70; 2004.

14. Moraes R. Análise de conteúdo. Educação. 1999; 22(37):7-32.

15. Loguércio RQ, Del Pino JC. Os discursos produtores da identidade docente. Ciência \& Educação. 2003; 9(1):17-26.
16. Gouvea G, Sanchez C, Machado MAD, Monteiro R, Silveira G, Santos JF. O ensino de Ciências na escola básica e a universidade: considerações sobre formação inicial e continuada de professores. RBPG. 2012; 2(8):395-417.

17. Diniz R, Campos L. Formação inicial reflexiva de professores de ciências e biologia: possibilidades e limites de uma proposta. RBPEC. 2011; 4(2):27-39.

18. Pacheco JA. Formação inicial e continuada: a articulação fragmentada. Educação e Cidadania. 2009; 11(11):85-98.

19. Lovisolo H. Educação física: arte da mediação. Rio de Janeiro: Sprint; 1995.

20. Darido SC, Rangel-Betti IC, Ramos GNS, Galvão Z, Ferreira LA, Mota e Silva EV, et al. Educação Física, a formação do cidadão e os parâmetros curriculares nacionais. Revista Paulista de Educação Física 2001; 15(1):17-32.

21. Copetti J, Soares RG, Puntel RL, Folmer V. Conhecimento dos professores de Educação Física para abordagem do tema saúde em suas aulas. R bras Ci. e Mov. 2012; 20(4):26-33.

22. Mohr A. A natureza da educação em saúde no ensino fundamental e os professores de ciências [tese]. Florianópolis: Universidade Federal de Santa Catarina; 2002.

23. Rufino LGB, Darido SC. Educação física escolar, tema transversal, saúde e livro didático: possíveis relações durante a prática pedagógica. $\mathrm{R}$ bras $\mathrm{Ci}$. e Mov. 2013; 21(3):21-34.

24. Catalan VC. La transversalidad y la escuela promotora de salud. Rev Esp Salud Pública. 2001; 75(6):505-16.

25. Azambuja CR, Brum LM, Carpilovsky CK, Cureau FV, Duarte PM, Santos DL, et al. Prioridades em saúde de escolares do ensino médio. ConScientiae Saúde. 2013; 12(2):185-94.

26. Leonello VM, L'abbate S. Educação em saúde na escola: uma abordagem do currículo e da percepção de alunos de graduação em pedagogia. Interface. 2006; 10(19):149-66.

27. Eichler ML, Del Pino JC. A produção de material didático como estratégia de formação permanente de professores de ciências. REEC. 2010; 9(3):633-56.

28. Lara S, Copetti J, Lanes KG, Puntel RL, Folmer V. Imagem corporal, medidas antropométricas e atividade física: ferramentas para a educação em saúde na escola. Rev Ciências \& Ideias. 2013; 4(2):1-22.

29. Lara S, Salgueiro ACF, Lara M, Puntel RL, Folmer V. Educação e saúde no contexto escolar: saúde cardiovascular como tema gerador no curso normal médio. REEC. 2013; 12(1):167-90.

30. Lanes KG, Lanes DVC, Puntel RL, Soares FAA, Folmer, V. Sobrepeso e obesidade: implicações e 
alternativas no contexto escolar. Rev Ciências \& Ideias. 2011; 3(1):1-18.

\section{CONFLITO DE INTERESSES}

Os autores declaram não haver conflitos de interesse.

\section{AUTOR PARA CORRESPONDÊNCIA}

Cati Reckelberg Azambuja

cati.razambuja@hotmail.com

Submetido em 19/06/2016

Aceito em 19/07/2016 